(Página deixada propositadamente em branco) 
MARIA PAULA SERRA DE OLIVEIRA

Coordenadora

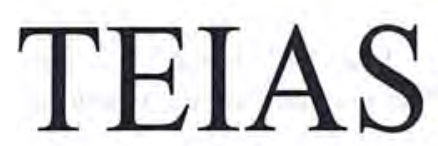

MATEMÁTICAS

Frentes na Ciência e na Sociedade 
(C) Gradiva - Publicações, L. da / Imprensa da Universidade de Coimbra, 2004 Coordenação editorial: Maria Paula Serra de Oliveira

Tradução: Artur Soares Alves

Carlota Isabel Leitão Pires Simões

Francisco José Craveiro de Carvalho

João Filipe Cortez Rodrigues Queiró

José Miguel Dordio Martinho de Almeida Urbano

Lia Sandra dos Santos

Mário da Silva Rosa

Paulo Eduardo Aragão Aleixo Neves de Oliveira

Revisão do texto: Isabel Pedrome

Capa: António Barros [Imprensa da Universidade. Coimbra], com imagem de

E. M. de Melo e Castro, "Fract 010 explod MC", Dezembro de 2003

[Fractal original gerado no Fractint com tratamento no Photoshop 7.0]

Infografia: Estúdios Estímulus [design]

Paginação: António Resende e Victor Hugo Fernandes

Impressão e acabamento: G.C. - Gráfica de Coimbra, L. da

Reservados os direitos para Portugal por:

Gradiva - Publicações, L. ${ }^{\text {da }}$ e Imprensa da Universidade de Coimbra

Gradiva - Publicações, L. ${ }^{d a}$

Rua Almeida e Sousa, 21, r/c, esq. • 1399-041 Lisboa

Telefs. $213974067 / 8 \cdot 213971357 \cdot 213953470$

Fax $213953471 \cdot$ Email: gradiva@ip.pt

URL: http://www.gradiva.pt

Imprensa da Universidade de Coimbra

Rua Antero de Quental, 195 • 3000-033 Coimbra

Telefs. 351239853110

Fax 3512398531 19 e-mail: fjrpress@ci.uc.pt

URL: http://www.imp.uc.pt

ISBN: 972-662-970-5

1." edição: Maio de 2004

Depósito legal n. ${ }^{\circ} 210431 / 04$

OBRA PUBLICADA COM O PATROCÍNIO DE:

CENTRO DE MATEMÁTICA DA UNIVERSIDADE DE COIMBRA DEPARTAMENTO DE MATEMÁTICA DA UNIVERSIDADE DE COIMBRA

FCT Fundação para a Ciência e a Tecnologia

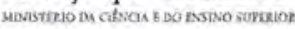


Alfio Quarteroni

École Polytéchnique Fédérale de Lausanne, Suiça

Politécnico de Milão, Itália

\section{Modelação matemática: uma síntese entre a teoria e as aplicações ${ }^{1}$}

\section{INTRODUÇÃo}

O objectivo deste texto é analisar a modelação matemática, o seu papel na ciência da engenharia bem como em ambientes não académícos.

Nas últimas décadas testemunhámos um enorme aumento da utilização da matemática no desenvolvimento teórico de diversos campos científicos bem como em aplicações nos mais variados aspectos da vida real.

Há cerca de meio século, salvo raras excepções, a expressão «matemática aplicada» significava essencialmente a aplicação da matemática à mecânica. Hoje em dia a matemática pode ser considerada um elemento da aprendizagem e do processo descritivo de inúmeras disciplinas como, por exemplo, a física, a química, a biologia, a engenharia, a medicina e a economia.

A modelação matemática é um processo que pretende resolver problemas provenientes destas disciplinas, ou seja, a sua representação por um conjunto de equações matemáticas, a análise das suas propriedades qualitativas, o estabelecimento de métodos numéricos para a sua

${ }^{1}$ Tradução de Lia Sandra dos Santos. 
aproximação e, finalmente, a sua implementação em computador através de algoritmos apropriados (figura 1).

Podemos questionar-nos sobre o que motiva a utilização da modelação matemática, que beneficios se podem obter com a sua utilização. No tocante às ciências experimentais, elas recorrem cada vez mais à utilização de ferramentas matemáticas sofisticadas, o que lhes permite evoluir de um estado empírico inicial para uma estrutura logicamente organizada. As motivações de outros tipos de ciências podem, no entanto, ser de natureza diferente.

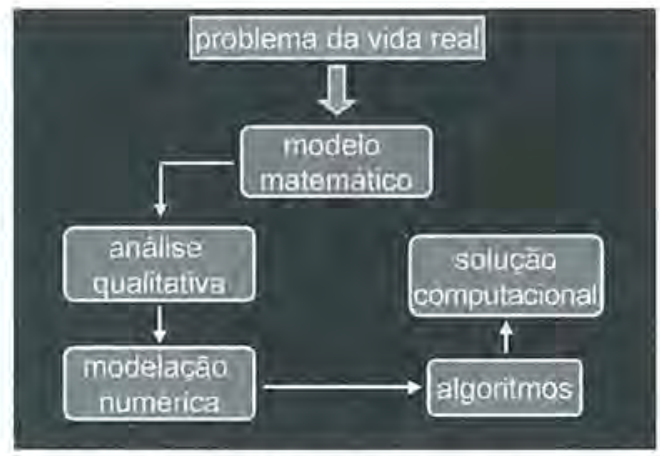

Fig. I-Modelaçăo matemática

Podem citar-se inúmeros casos de utilização de modelos matemáticos para simular ou mesmo controlar dinàmicas sociais ou demográficas. Em muitos dos problemas encontrados na economia, os modelos matemáticos fornecem informações quantitativas, operando com um número de variáveis desconhecidas consideravelmente maior do que aquele que poderia ser tido em consideração no contexto de uma análise qualitativa. Isto acontece, por exemplo, sempre que desejamos prever a reacção de sistemas fortemente interactivos quando as condições de referência (i.e., o mercado) se alteram muito rapidamente. Por último, deve ser referido que foi dado um forte impulso à utilização de modelos matemáticos em grande escala, pela análise sistémica, através da qual o campo de observação foi alargado para uma escala global. Um exemplo significativo é o modelo, criado durante o período da Guerra Fria, para a previsão do comportamento da atmosfera após a utilização de armas nucleares, perante a terrivel perspectiva do inverno nuclear. Outro modelo é aquele que procura prever a alteração atmosférica global, envolvendo a interacção 
entre os oceanos, a terra e a atmosfera, e cujo objectivo é a simulação precisa das variações climáticas devidas ao efeito de estufa.

\section{A MODELAÇÃo MATEMÁTICA NA CULTURA DA ENGENHARIA}

Através da modelação matemática, um problema proveniente do mundo real é transferido do seu universo próprio para um habitat onde pode ser correctamente analisado e resolvido numericamente, sendo depois transferido de novo para o seu contexto de origem para visualização, análise e validação dos resultados obtidos.

Um modelo não expressa necessariamente a verdadeira natureza do problema nem a sua complexidade, pois a realidade é muitas vezes demasiado complexa, não sendo possível a sua rigorosa representação em termos de fórmulas matemáticas. Contudo, o modelo tem de fornecer uma síntese útil do problema.

Uma característica da modelação é a abstracção, isto é, a capacidade de identificar características comuns em campos diferentes, permitindo a elaboração, a priori, de ideias muito gerais, e depois a sua aplicação em contextos muito diferentes uns dos outros.

Muitos ramos da engenharia, tais como a aeronáutica, a mecânica, a engenharia civil, a quimica e a engenharia nuclear têm tradicionalmente beneficiado com a utilização de modelos matemáticos para o planeamento, controlo, optimização e gestão de processos industriais e tecnológicos. Mais recentemente, os modelos matemáticos foram também introduzidos noutros campos, de entre os quais mencionamos, sem ser exaustivos, a microelectrónica, o ambiente e as finanças (vide figura 2).

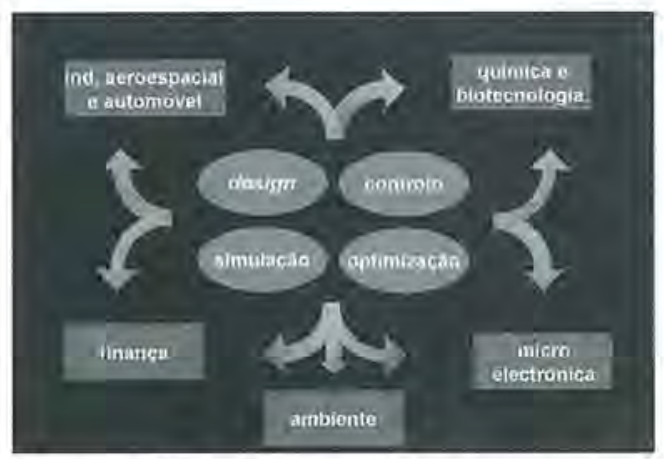

Fig. 2 -Objectivos dos modelos matemáticos e campos de aplicação 
Os engenheiros utilizam a análise experimental e a simulação numérica de forma complementar. A última é mais flexível no que diz respeito à variação de parâmetros físicos ou à mudança das condições de limite e às formas geométricas. A simulação numérica pode fornecer uma descrição pontual de todas as variáveis que intervêm no processo em questão.

A modelação matemática é por isso um elemento de ligação entre a análise experimental e o planeamento da produção (vide figura 3 ). No topo deste processo, os modelos matemáticos são sempre inspirados por princípios físicos fundamentais, tais como as equações do equilíbrio estático, ou os princípios da massa, energia e o momento de conservação da dinâmica do meio contínuo, sendo a descrição da reologia dos materiais, a determinação de condições de limite e a dimensionalização e medições dos coeficientes do problema e parâmetros descritivos fornecidos pela análise experimental.

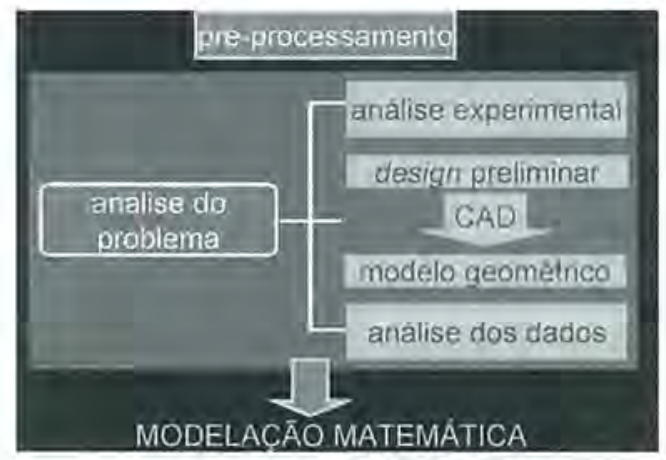

Fig. 3-Análise preliminar

Muitas vezes, uma característica adicional do modelo matemático é a apresentação de um modelo geométrico, isto é, a representação por sólidos modeladores ou ferramentas $\mathrm{CAD}$ da região tridimensional complexa na qual as equações do modelo devem ser resolvidas.

$\mathrm{Na}$ sequência deste processo, a complexidade dos campos de vectores tridimensionais obtidos tem de ser analisada de uma forma logicamente organizada e validada à luz dos dados experimentais disponiveis, e principalmente da intuição da engenharia. Esta análise retrospectiva, por 
sua vez, conduz a um processo iterativo que permite modificar o modelo, as suas equações ou os seus parâmetros, até que os resultados numéricos obtidos estejam de acordo com as verificações experimentais para uma grande família de casos de teste (vide figura 4).

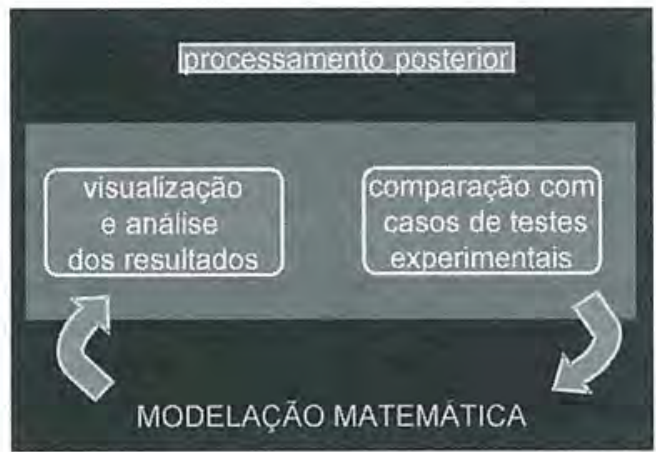

Fig. 4-Análise a posteriori

O exemplo ilustrado na figura 5 refere-se ao projecto de um veículo (um carro ou um avião) e mostra a interacção entre os testes no túnel de vento e a simulação numérica, antes de se obter o projecto final.

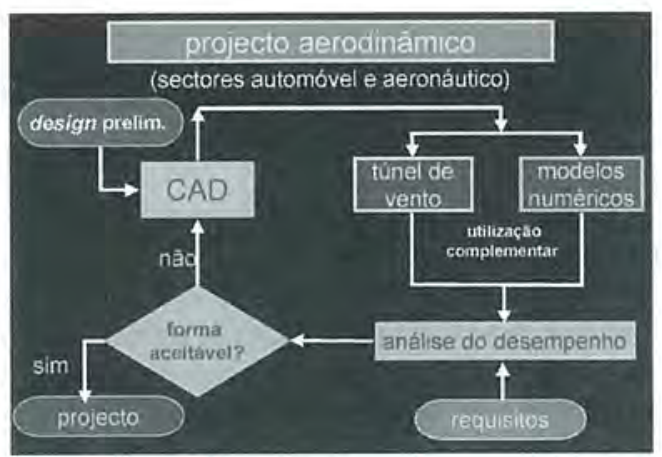

Fig. 5-Da concepção inicial à final 


\section{A SIMULAÇÃo NUMÉricA}

Para um especialista de matemática aplicada, o principal objectivo é a resolução efectiva do problema expresso pelo modelo matemático. Muitas vezes, estes problemas são determinados por complexas equações de derivadas parciais, cuja solução explícita não é conhecida ou cuja representação não é passível de construção. Os algoritmos numéricos têm então de ser desenvolvidos de forma a permitir sempre a solução do modelo em questão, desde que este esteja bem formulado do ponto de vista matemático.

A simulação numérica do modelo matemático visa garantir que a solução aproximada seja calculada computacionalmente de modo eficiente e que o erro entre a solução física e numérica seja pequeno e controlável.

A eficiência do algoritmo é medida, entre outros factores, pela sua complexidade, isto é, a quantidade de recursos necessários para implementar o algoritmo no computador, nomeadamente o tempo de computação e a memória requerida. A este propósito, note-se que os computadores disponiveis hoje em dia não permitem a solução de problemas de complexidade arbitrária.

Considerem-se alguns exemplos provenientes de sistemas determinísticos cujo comportamento é descrito por equações de derivadas parciais da dinâmica dos fluidos. Esta disciplina é de um interesse primordial, não só porque se encontra numa larga variedade de aplicações, mas também porque dá origem a um conjunto de problemas que estão entre os mais dificeis de resolver de toda a matemática aplicada.

\section{UM EXEMPLO INTERESSANTE: MODELAÇÃO NA DINÂMICA DOS FLUIDOS}

Os fluidos têm uma enorme importância na vida quotidiana. De facto, a dinâmica da atmosfera, a dispersão dos poluentes no ar, a formação de correntes e a circulação de sedimentos em cursos de água, a corrente sanguínea no nosso sistema cardiovascular são problemas de dinâmica dos fluidos. Outros processos de natureza aparentemente diferente podem também ser descritos por modelos de fluidos. Por exemplo, a nível microscópico, os electrões num meio semicondutor comportam-se como um fluido condutor de corrente.

Todos os exemplos precedentes, bem como muitos outros que se poderiam citar, podem ser modelados por um sistema de equações de 
derivadas parciais introduzido pelo engenheiro francês Louis Marie Henri Navier e pelo físico irlandês Sir George Gabriel Stokes. Note-se que apesar de estas equações serem conhecidas há mais de um século, muitos aspectos do comportamento dos fluidos escapam ainda à nossa compreensão.

Os métodos analíticos podem proporcionar uma forma explícita de solução para as equações de Navier-Stokes, no caso de serem consideradas hipóteses tão restritivas que o problema simplificado tenha pouco interesse prático.

A tendência dos fluidos para mostrar um comportamento complexo ou, mais precisamente, turbulento é a razão para tal difículdade. A propósito, a turbulência não é uma propriedade constitutiva (ou intrínseca) do fluido; é, sim, um regime especifico do escoamento do fluido que surge sempre que um grande número de graus de liberdade intervém na dinâmica dos fluidos. Tratando-se de um modelo não-linear, todas as escalas de movimento possível interagem. Tal interacção é responsável pela turbulência.

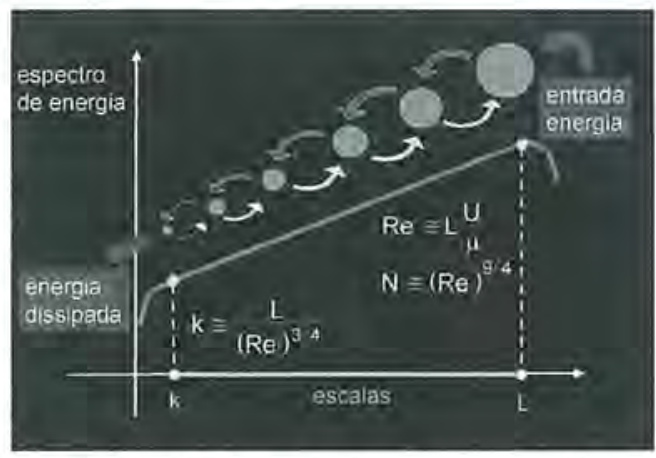

Fig. 6-Escalas de turbulência e espectro de energia

Um indicador do nível de turbulência é o chamado número de Reynolds, directamente proporcional à velocidade média do fluido $\mathrm{e}$ inversamente proporcional à sua viscosidade molecular. $\mathrm{O}$ número de Reynolds expressa a importância da convecção que ocorre em escalas macroscópicas, relativamente à dissipação, que ocorre em vez disso a nível molecular.

A análise das equações de Navier-Stokes permite afirmar que o número de graus de liberdade de uma corrente turbulenta é da ordem de $N=\operatorname{Re}^{9 / 4}$ 
(por uma questão de simplicidade, podemos pensar que estamos a trabalhar em cada nível temporal com uma série de Fourier com $\mathrm{N}$ modos). Dado que o número de Reynolds de muitas correntes, por exemplo as que circundam num avião, é da ordem de $10^{6}$, o número de graus de liberdade correspondente pode ser maior que $10^{13}$, isto é, dez biliões.

A energia do fluido em grandes escalas, devido às condições de fronteira e às forças volúmicas, é transferida para escalas cada vez menores com interacção não-linear dos graus de liberdade activos no fluido, através de uma espécie de cascata de energia, baixando para uma escala tão pequena que a energia se dissipa no calor. A escala activa mais pequena, que indicaremos por K, é conhecida por escala de Kolmogorov e é directamente proporcional à escala maior e inversamente proporcional a $\operatorname{Re}^{3 / 4}$ (vide figura 6).

Por exemplo, na turbulência atmosférica, a que tem de ser simulada nas previsões metereológicas, o número de Reynolds é da ordem de $10^{8}$. Isto implica que a entrada de energia, na escala de um quilómetro, afecta até as escalas na ordem de um milímetro. Uma característica geral da turbulência do fluido é a presença das regiões de «escoamento coerente», com turbulência que se auto-organiza dando origem a padrões de escoamento regular e não caótico, espirais normalmente designadas por vórtices. As estruturas coerentes estão organizadas de forma hierárquica, no sentido em que os últimos vórtices geram vórtices ainda mais pequenos, e assim sucessivamente até um diâmetro $\mathrm{K}$ da ordem da escala de Kolmogorov, como ilustra a figura 6. Reciprocamente, todas as escalas são influenciadas por uma escala mais pequena num processo em cadeia interminável.

Quando utilizamos a simulação numérica quase nunca podemos ter em consideração todo o conjunto das escalas activas, desde a macroescala $\mathrm{L}$ até à escala $\mathrm{K}$ de Kolmogorov.

Por exemplo, na simulação da troca de energia até à escala de Kolmogorov, na turbulência atmosférica, deviamos utilizar um passo de discretização espacial da ordem de um milimetro. Isto implicaria resolver um sistema dinâmico com $10^{18}$ variáveis, sistema este que nenhum computador existente consegue tratar. É por isso necessário desistir da simulação numérica directa e utilizar métodos de redução que aproximam apenas um númeró limitado de escalas, mas fornecem um modelo conveniente da troca de energia de pequenas escalas e de grandes escalas, através de processos apropriados de cálculo da média e de normalização (vide figura 7 para uma representação qualitativa desta abordagem). 


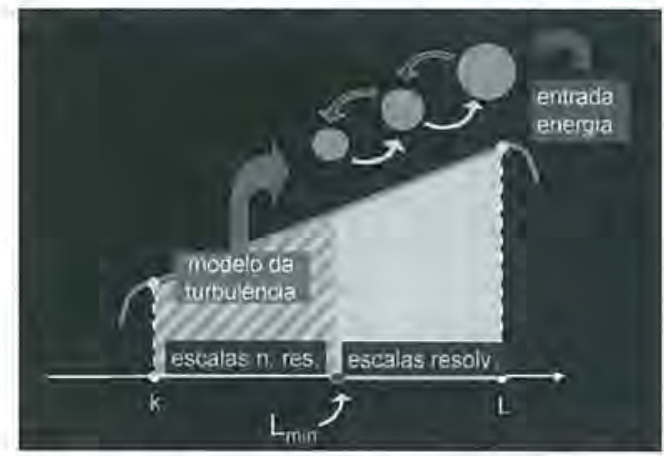

Fig. 7 - Escalas na simulação de escoamentos turbulentos

Uma outra dificuldade reside na modelação e simulação dos fluxos em presença de reacções quimicas, quer no caso de problemas de uma só fase quer no caso de problemas multifase. Por limitações de tempo não desenvolveremos este aspecto, apesar do interesse que assume em muitas aplicações. Na indústria, a utilização da experimentação constituiu durante muito tempo o único instrumento disponível para analisar os fenómenos relacionados com os escoamentos dos fluidos. Em alguns casos, no entanto, a temperatura e a velocidade envolvidas são tão elevadas que a experimentação em túneis de vento é mesmo impossivel, como acontece, por exemplo, na reentrada na atmosfera de um veículo espacial. Noutras situações, como por exemplo o estudo dos efeitos fisiopatológicos induzidos pela dinâmica do sangue, a experimentação in vivo, além de ser pouco precisa, é obviamente crítica para o paciente.

A modelação numérica do movimento dos fluidos, se for realizada com grande rigor matemático, pode fornecer resultados precisos para a compreensão de fenómenos complexos e ser um instrumento de apoio na análise experimental e na realização de projectos industriais. Naturalmente, é preciso desenvolver métodos numéricos adequados e utilizar algoritmos que explorem de modo óptimo as modernas arquitecturas de cálculo vectorial e paralelo.

\section{SIMPLIFICAR PARA RESOLVER}

A complexidade dos problemas a resolver pode ser, no entanto, demasiadamente elevada relativamente ao papel que a simulação numérica pode assumir. Por exemplo, na fase do projecto e optimização de um 
yeículo na indústria automobilística, recorre-se à utilização interactiva de diversos códigos de cálculo para analisar as várias componentes do projecto. Os tempos de elaboração necessários à análise de alguns elementos são muitas vezes demasiadamente elevados, não permitindo várias simulações diárias. Nestes casos impõe-se uma reformulação do modelo e a sua posterior simplificação. Por exemplo, o modelo reduzido das equações de Navier-Stokes em que se elimina a viscosidade dá origem às chamadas equações de Euler, suficientes para prever de modo preciso a pressão na aerodinâmica externa e também as trocas de energia interna entre fluido e máquina. Uma simplificação posterior conduz à equação do potencial não-linear, uma única equação e não um sistema de equações como anteriormente, que é adequada para descrever fluxos irrotacionais e isotrópicos, sendo por este motivo frequentemente utilizada para a simulação de regimes não ultra-sónicos.

É claro que a utilização de modelos simplificados diminui drasticamente a complexidade do problema, tornando assim possível efectuar simulações computacionais, que de outro modo não seriam realizáveis. Esta simplificação deve ser, no entanto, justificada: do ponto de vista fisico, não deve alterar o significado do problema em análise, e do ponto de vista matemático deve conservar as propriedades teóricas fundamentais do modelo original. A síntese destas duas exigências nem sempre é fácil e exige um esforço conjunto de matemáticos e engenheiros. Num outro âmbito, a redução da complexidade pode também obter-șe recorrendo a partições geométricas do problema, o que o torna particularmente adaptado à utilização do cálculo paralelo. Neste caso, transforma-se o problema numérico original numa sucessão de problemas de dimensão reduzida que podem ser resolvidos simultâneamente num ambiente de cálculo com multiprocessadores. Na figura 8 ilustra-se de modo esquemático o processo de simulação do fluxo que se gera nas vizinhanças da asa de um avião durante a aterragem.

$\mathrm{O}$ desenvolvimento de arquitecturas paralelas incentivou os matemáticos a estudar novos métodos de cálculo, baseando-se muitas vezes na reformulação do próprio modelo matemático.

Na figura 9 mostra-se que a diminuição do tempo de cálculo, devido a vinte anos de evolução no hardware, é inferior à diminuição resultante do progressivo aperfeiçoamento dos métodos numéricos, incluindo a sua adaptação às arquitecturas vectoriais.

O desenvolvimento em simbiose do hardware e do software é, sem dúvida, uma das condições determinantes para resolver com sucesso os modelos matemáticos de complexidade crescente que emergem nos desafios científicos contemporâneos. 


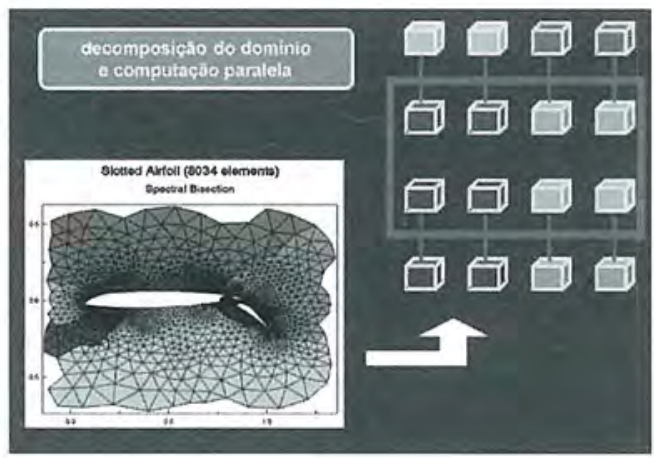

Fig. 8-Decomposição do domínio e cálculo paralelo

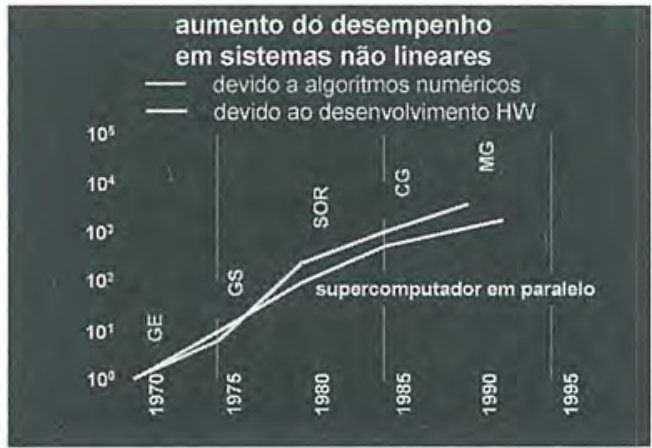

Fig. 9 - Diminuição do tempo de cálculo na resolução de sistemas lineares 


\section{$\begin{array}{llllll}\text { C I E } & \mathbf{N} & \mathbf{C} & \mathbf{A}\end{array}$}

mitiram realizar com sucesso tarefas tão distintas como a programação de um voo a Marte, a previsão de resultados eleitorais, a explicação do funcionamento de alguns mecanismos do sistema nervoso, ou a abordagem critica de obras de arte e de textos literários. Da ciência à sociedade, dos grandes avanços técnicos à solidez de uma argumentação lógica, a Matemática constrói teias de uma imensa flexibilidade resultante do carácter universal da sua linguagem.

Neste livro, personalidades de diferentes universos dão o seu testemunho sobre a forma como usam as teias matemáticas para tecer a sua própria visão do mundo.

Maria Paula Serra de Oliveira é professora de Matemática na Faculdade de Ciências e Tecnologia da Universidade de Coimbra. 